\title{
The Teaching of Chinese Abstract Words Related to the Scale of Reasonableness \\ Ruifeng Wang
}

College of Advanced Chinese Training, Beijing Language and Culture University, Beijing, China wangruifeng@blcu.edu.cn

Keywords: Scale; The scale of reasonableness; Abstract words; The teaching of abstract word

Abstract. Chinese abstract words related to the scale of reasonableness can be divided into two types. One is the abstract words being used to connect the reasonable events of reference points, the other is being used to connect reasonable events of target points, and both of them not only have their own features but also have co-occurrences and collocations in language expressions. Therefore efficient teaching for Chinese abstract words can be designed from this perspective.

\section{情理值梯级虚词的教学 \\ 王瑞烽 \\ 北京语言大学汉语进修学院, 中国 北京 100083 \\ wangruifeng@blcu.edu.cn}

摘要: 情理值梯级虚词可分为连接 “参照点情理值事件” 的虚词、连接 “目标点情理值事件” 的虚词两大类, 它们在语言表达中还常存在着共现和搭配。从情理值梯级的角度可以对之进 行有效的教学设计。

关键词: 梯级; 情理值梯级; 虚词; 虚词教学

\section{1. 引言}

西方语言学者 [1］［2］［3］４4］［5］［6］［7］发现人类的很多基本概念域都是以梯级（scale） 形式存在的, 梯级表现出程度和等级的特性, 在言语交际中, 概念域中的这种梯级可以帮助 人们通过梯级推理（scalar inference）获得隐含的梯级含义 (scalar implicature)。梯级 推理是指人们以梯级中的某一参照点的情况去推知或者认定同一梯级中的目标点的情况, 其 推导方法分为最基本的两种: 从较低级的参照点的情况推导出较高级的目标点的情况; 从较 高级的参照点的情况推导出较低级的目标点的情况。

张旺喜 [8]指出 “语义理解的基础是一个涉及背景知识的复杂认知结构。这种复杂的认知结构 反映着特定社会各种相关认知域里的文化环境中的说话人对某个或某些领域里的经验具有统 一的、典型的、理想化的理解。” 这是一种 “理想化的认知模型 (ICM)”，符合 “理想化的认 知模型” 的事件一般是自然世界和现实生活中常态的、符合一般情理的事件，这样的事件具 有较高的 “情理值”，如 “大力士搬得动”、“小孩儿搬不动”、“冬天气温低”、“夏天气温高”; 而不符合 “理想化的认知模型” 的事件一般是自然世界和现实生活中特殊的、不符合一般情 理的事件，这样的事件具有较低的 “情理值”，如 “大力士搬不动”、“小孩儿搬得动”、“冬天 气温高”、“夏天气温低”。

汉语中的很多虚词都与情理值梯级相关, 从情理值梯级的角度对之进行研究, 可以获得新的 认识, 并可以为这些情理值梯级虚词的教学提供启示。

\section{2. 情理值梯级虚词}


情理值梯级存在于人类的认知结构中, 同时它也会反映到人类的言语交际中, 在语言体系中, 也会有很多语法结构与梯级密切相关。国内英语学界的一些学者 [9] [10] [11]在西方梯级含义 理论的启示下对于外语和汉语中的梯级含义现象进行了一定的研究, 研究内容涉及极性词语 的梯级含义功能、极性隐喻现象、夸张性隐喻的梯级含义功能、虚拟参照点的梯级含义功能、 主观量的梯级模型解释等。汉语学界的很多学者也从梯级的角度对于汉语语法展开了研究, 发现很多句式和虚词都与梯级密切相关, 从梯级的角度分别对之进行了更准确和深入的分析。 需要说明的是, 在研究中有的学者直接使用了梯级的概念, 而有的学者使用了其他的术语, 如预设集合、等级尺度、隐性量级序列、隐性语义等级序列、语用量级等，但其所指其实都 是梯级。已研究的关涉梯级的虚词主要有：“连” [7] [12] [13] [14]、“何况” [15] [16]、“别 说” [17][18]、“即使” [19][20]。

我们可以把陈述的情理值事件看作是进行梯级推理的参照点, 是 “参照点情理值事件”, 而被 推理的情理值事件是梯级推理的目标点, 是 “目标点情理值事件”。那么, 汉语中与情理值事 件梯级推理相关的虚词则主要可以分为两类：接 “参照点情理值事件” 的虚词和连接 “目标 点情理值事件” 的虚词。连接 “参照点情理值事件” 的虚词主要有: “尚且、连、都/也、即 使、就是”，连接“目标点情理值人或物”的虚词主要有：“何况、别说”。

由于这些虚词常常都关涉梯级，所以它们往往都可以一起共现或者互相搭配，这种共现和搭 配是丰富多样的，如 “别说” 可以与 “就是”、“即使”、“连” 等情理值梯级虚词搭配，“连” 可以与 “何况”、“尚且”、“别说” 等搭配, “即使” 可以与 “别说”、“何况”、“尚且”等搭配。

\section{3. 情理值梯级虚词的教学}

基于前文对关涉情理值梯级的虚词的研究，对于这类虚词的教学，我们提出以下教学建议:

（1）注意教学的层次性。首先要使留学生理解情理值梯级, 可以通过形象、贴近学生生活的 例句列举情理值高低不同的多种情况, 特别是情理值高低差异比较大、比较明显的情况, 引 导学生认识到情理值存在高低之分。例如:

$\begin{array}{ccccc}\text { (这个箱子) } & \text { (这个箱子) } & \text { (这个汉字) } & \text { (这个汉字) } & \text { 情 } \\ \text { 大人搬得动 } & \text { 小孩儿搬不动 } & \text { 中国人认识 } & \text { 外国人不认识 } & \text { 理 } \\ & & & \text { 值 }\end{array}$

\section{小孩儿搬得动 大人搬不动 外国人认识 中国人不认识}

通过这些例句，使用提问的方式引导学生认识情理值梯级。如，“大人的力量大还是小孩儿的 力量大? ”、“谁更可能搬得动这个箱子, 大人还是小孩儿? ”, 通过这样的方式, 使学生认识 到, 比较而言, 上面的事件出现的可能性比较大, 是普通的、常规的事件, 而下面的事件出 现的可能性比较小，是特殊的、反常的事件。

使用提问的方式引导学生认识情理值梯级。如，“大人的力量大还是小孩儿的力量大? ”、“谁 更可能搬得动这个箱子, 大人还是小孩儿? ”, 通过这样的方式, 使学生认识到, 比较而言, 上面的事件出现的可能性比较大, 是普通的、常规的事件, 而下面的事件出现的可能性比较 小, 是特殊的、反常的事件。在此基础上, 再引导学生自己说出其他情理值高低不同的几组 事件, 加强学生对于情理值梯级的理解。

其次, 使学生理解梯级推理的两种基本方式。首先通过例句, 使学生理解两个情理值事件的 对比推理, 即通过情理值较小的事件来推理情理值相对较高的事件的情况。如: “这个汉字连 中国人都不认识, 何况外国人呢? ”、“这个汉字连外国人都认识, 何况中国人呢? ”, 最后, 使学生理解全量推理, 即通过极端情理值事件来推理所有情理值事件的情况。如: “这个汉字 连三岁小孩都会写, 谁不认识呢？”、“这个汉字连汉字专家都不认识, 谁还能认识呢？”。 
（2）在教学中, 为了使教学简明、易于理解, 不强调句子中肯定的低情理值事件还是否定的 高情理值事件, 而是直接将表述的事件整体看作是一个事件, 都是自然世界和现实生活中特 殊的、不符合一般情理的事件，是发生可能性小的事件。

（3）要使学生理解情理值推理的作用。说话人为了支撑自己对于 A 事件的观点, 不是直接表 述 $\mathrm{A}$ 事件的情况, 而是通过表述情理值相对较小的 B 事件的情况来间接地使得 $\mathrm{A}$ 事件的情况 不言而喻, 更加确定。也就是说, 说话人是为了让自己的观点更加立得住, 不可辩驳。

(4) 例句和练习的编写要慎重考虑诸多因素。第一, 要注意文化预设因素。由于留学生来自 不同的国家、文化圈, 应该以人类普遍共有的认知经验为基础编写, 以便于留学生理解。外 部世界的共同点反映到人类的头脑中, 形成了普遍的认知经验, 如生老病死的感受及必然性、 饥渴困累的感受及解决办法。这些都是人类同有的普遍认知经验。但由于自然环境、文化等 因素的影响, 人的认知经验还具有社团性, 如在汉族人的认知经验中, 过春节要团圆、红色 代表喜庆等。

认知语言学认为, 在语言交际中, 语义理解和表达都会离不开人的认知经验的参与, 认知经 验是言语交流的前提、基础。以 “即使” 为例, 如 “他即使每天吃很多也不长胖。”、“他即使 春节的时候也不回家。”, 第一个句子中的预设一一吃很多会长胖, 这是人类普遍共有的认知 经验, 说话人把这种认知经验作为预设隐含在句子中, 而这种认知经验是不同种族、国家、 文化圈的人都共有的，所以这句话对于所有的人都是可理解的。而第二个句子中的预设- 春节要回家团圆, 只是了解汉文化圈习俗的人所拥有的认知经验, 对于不拥有这种认知经验 的人来说, 是很难理解的。如果例句的预设涉及到留学生所不具备的认知经验, 就会对留学 生的理解造成困难。这一点应该特别注意, 应该审视例句和练习的预设与留学生的认知经验 是否相符。

第二, 要注意个人主观性因素。符合 “理想化的认知模型 (ICM)” 的情理值梯级是特定社会 各种相关认知域里的文化环境中的人们对某个或某些领域里的经验所具有统一的、典型的、 理想化的理解。这样的情理值梯级是具有社团规约性的。

有时说话人所认为的情理值梯级只是个人或某些人对世界中事物、事件的独特知识和看法, 不是整个社团普遍知道和认同的。这种情理值梯级则是具有个人主观性的。如: “他连小王都 赢不了, 何况我呢? ”在例句和练习编写时, 应该避免涉及到具有个人主观性的情理值梯级, 特别是在语法引入阶段。

（5）在教学中注意句式中虚词搭配格式的丰富性。在汉语教材中, 不论是初级教材, 还是 中高级教材, 对于某一虚词的讲解, 大都是给出某一常见搭配格式, 如在讲解 “何况” 时, 一般只是给出 “尚且 ……何况 ……”的搭配格式。

但通过语料库调查会发现, 与 “何况” 搭配的格式中, 这一格式其实并不是出现频率最高的 格式, 其中 “都/也……何况……” 的使用频率更高, 其他搭配格式如 “连……都/也……何 况……” “即使……都/也……何况……” 也有较高的使用频率。我们在教学中不能忽略这 些格式的教学, 特别是在中高级阶段。

\section{4. 结语}

汉语中的很多虚词都与情理值梯级相关, 除了文中提到的虚词, 还有那些虚词关涉着情理值 梯级? 这些虚词相互之间的区别与特质是什么? 如何从情理值梯级的角度进行科学、合理的 教学设计? 这些问题值得进一步全面而深入地进行研究。

\section{5. 致谢}

本成果受北京语言大学校级科研项目 (中央高校基本科研业务专项基金) 资助, 项目编号为 
13YBG09。

\section{参考文献}

[1] Ducrot, O. La Prevue et. Le Dire [M]. Paris: Maison Mame, 1973.

[2] Levinson, S. C.. Pragmatics [M].Cambridge: Cambridge University Press, 1983.

[3] Israel, M. The Rhetoric of Grammar: Scalar Reasoning and Polarity Sensitivity [D]. U. C. San Diego, 1998.

[4] Israel, M. Minimizers, maximizers and the rhetoric of scalar reasoning [J]. Journal of Semantics 2001(18): 297-331.

[5] Carston, R. Thoughts and Utterances:The Pragmatics of Explicit Communication[M]. Oxford: Blackwell, 2002.

[6] Israel, M. The pragmatics of polarity [A]. In L. R. Horn \& G. Ward (eds.). The Handbook of Pragmatics [M]. Blackwell Publishing, 2004:701-723.

[7] Fauconnier, G. Pragmatic Scales and Logical Structure. Linguistic Inquiry [J], 1975,Vol. VI, No. 3: 353-375.

[8] 张旺喜.连字句的序位框架及其对条件成分的映现 [J] . 《汉语学报》,2005 年,第 2 期:3-14.

[9] 蒋勇.夸张性隐喻的梯级含义功能[J], 《现代外语》,2004 年,第 3 期:274-281.

[10]龚卫东.广义梯级含义理论及其应用[D], 上海外国语大学博士学位论文,2006 年.

[11]周家发.主观量的梯级模型解释[J]，《汉语学习》,2012 年,第 4 期：29-38.

[12]刘丹青.作为典型构式句的非典型“连”字句[J]. 《语言教学与研究》,2005 年,第 4 期:1-12.

[13]袁毓林.试析“连”字句的信息结构特点 [J].《语言科学》,2006 年,第 2 期:14-28.

[14]石慧敏、吴为善.隐性语义等级序列的激活机制及其语篇整合效应[J].《世界汉语教学》,2014 年,第 4 期:449-460.

[15]张邱林.“尚且 p，何况 q”反逼句式[J].《世界汉语教学》,2009 年,第 3 期:343-354.

[16]陆方吉、李晓琪.“何况”的主观性表达一一兼析与“况且”的区别 [J].《汉语学习》,2013 年, 第 6 期: 47-54.

[17]张滟.构式“XAY let alone B”与“X(连)A 都/也 Y, 更不用说/别说 B”的语义一句法界面研究 一基于“交互主观性”认知观 $[\mathrm{J}]$. 《中国外语》,2010 年,第 1 期:38-46.

[18]周莉.现代汉语“别说”的语义、功能研究[D].吉林大学博士学位论文,2012 年.

[19] 戴悉心.“即使”句的分类及其语义语用分析[J].《世界汉语教学》,2001 年,第 2 期:49-53.

[20]王瑞烽. 从梯级的角度阐释"即使"句式及教学建议 [C]. 《汉语应用语言学研究》, 北京: 商务 印书馆,2015 年

\section{Acknowledgement}

This research was financially supported by Science Foundation of BLCU (Supported by "the Fundamental Research Funds for the Central Universities"), 13YBG09.

\section{References}

[1] Ducrot, O. La Prevue et. Le Dire [M]. Paris: Maison Mame, 1973. 
[2] Levinson, S. C.. Pragmatics [M].Cambridge: Cambridge University Press, 1983.

[3] Israel, M. The Rhetoric of Grammar: Scalar Reasoning and Polarity Sensitivity [D]. U. C. San Diego, 1998.

[4] Israel, M. Minimizers, maximizers and the rhetoric of scalar reasoning [J]. Journal of Semantics 2001(18): 297-331.

[5] Carston, R. Thoughts and Utterances: The Pragmatics of Explicit Communication [M]. Oxford: Blackwell, 2002.

[6] Israel, M. The pragmatics of polarity [A]. In L. R. Horn \& G. Ward (eds.). The Handbook of Pragmatics [M]. Blackwell Publishing, 2004:701-723.

[7] Fauconnier, G. Pragmatic Scales and Logical Structure. Linguistic Inquiry [J], 1975, Vol. VI, No. 3: 353-375.

[8] ZHANG Wangxi. The Sequential Framework of the Lian-sentence and the Projection of Conditional Constituent [J].Chinese Language Learning, 2005 (2):3-14.

[9] Jiang Yong. The Scalar Implicature Function of Hyperbolic Metaphor [J].Modern Foreign Language, 2004(3):274-281.

[10] Gong Weidong. The Extended Theory of Scalar Implicature and Its Applications [D]. A Dissertation of Shanghai International Studies University, 2006.

[11] Chow Kafat. A Scalar Model Interpretation for Subjective Quantity [J], Chinese Language Learning, 2014(4):29-38.

[12] Liu Danqing. The Atypical Lian-Clause as a Typical Construction [J], Language Teaching and Linguistic Studies, 2005(4):1-12.

[13] Yuan Yulin. The Information Structure of the Lian-construction in Mandarin [J], Linguistic Sciences, 2006(2):14-28.

[14] Shi Huimin, Wu Weishan. Activation Mechanism of the Covert Sematic Hierarchy and Its Effect on Discourse Integration [J], Chinese Teaching in the World, 2014(4):449-460.

[15] Zhang Qiulin, The Retrospective Sentence Pattern "Shangqie p, Hekuang q" [J], Chinese Teaching in the World, 2009(3):343-354.

[16] Lu Fangzhe, Li Xiaoqi, The Subjectivity Expression Function of "Hekuang"[J], Chinese Language Learning,2013(6):47-54.

[17] Zhang Yan, A Semantics-syntax Interface Study of a Constructions "XAY let alone B" and "X (lian) A dou/ ye Y, geng buyong shuo/bie shuo B"- Based on Cognitive Intersubjectivity [J], Foreign Languages in China, 2010(1):38-46.

[18] Zhou Li, A Study on "Bieshuo" in Modern Chinese: from Semantic and Functional Perspective [D], A Dissertation of Jilin University, 2012.

[19] Dai Xixin, A Classification and A Sematic Pragmatic Analysis of Jishi-sentence[J], Chinese Teaching in the World,2001(2):49-53.

[20] Wang Ruifeng, A Scalar Perspective Analysis and Teaching of Jishi-sentence[A], In Research on Chinese Applied Linguistics[C], Beijing: The Commercial Press,2015.

作者简介: 王瑞烽 (1977一), 男, 山东, 副教授, 主要研究方向为虚词教学、二语习得, blcuwang@126.com。 